\title{
Efficacy and Safety of TJP-008 Compared to 2 L PEG with Ascorbate in Colon Cleansing: A Randomized Phase 3 Trial
}

\author{
Ja Seol Koo', Jeong-Sik Byeon², Bo-In Lee ${ }^{3}$, Sung-Ae Jung ${ }^{4}$, Tae II Kim, and Yoon Tae Jeen ${ }^{6}$ \\ ${ }^{1}$ Division of Gastroenterology and Hepatology, Department of Internal Medicine, Ansan Hospital, Korea University College of Medicine, \\ Ansan, ${ }^{2}$ Department of Gastroenterology, University of Ulsan College of Medicine, ${ }^{3}$ Division of Gastroenterology, Department of \\ Internal Medicine, College of Medicine, The Catholic University of Korea, ${ }^{4}$ Department of Internal Medicine, Ewha Womans University \\ College of Medicine, ${ }^{5}$ Department of Internal Medicine, Yonsei University College of Medicine, and ${ }^{6}$ Division of Gastroenterology and \\ Hepatology, Department of Internal Medicine, Anam Hospital, Korea University College of Medicine, Seoul, Korea
}

\section{Article Info}

Received February 25, 2021

Revised May 7, 2021

Accepted June 14, 2021

Published online November 23, 2021

\section{Corresponding Author}

Yoon Tae Jeen

ORCID https://orcid.org/0000-0003-0220-3816

E-mail ytjeen@korea.ac.kr
Background/Aims: Polyethylene glycol (PEG)-based bowel preparations are effective cleansing agents for colonoscopy. However, they require relatively large volumes to be used even with agents such as 2 L PEG with ascorbate (2LPEG). This phase 3, randomized, single-blinded, multicenter, parallel-group study compared the efficacy of $1 \mathrm{~L} \mathrm{PEG}$ with high-dose ascorbate, TJP-008, to 2LPEG.

Methods: Patients undergoing colonoscopy were randomized (1:1:1) to receive TJP-008 as 1-day split dose (TJP-008-1) or 2-day split dose (TJP-008-2) regimen or to receive 2LPEG as a 2-day split dose regimen. Cleansing efficacy was evaluated using the Harefield Cleansing Scale. The primary endpoint was overall bowel cleansing success. Full analysis set (FAS) and per protocol set (PPS) analyses were performed.

Results: Of the 314 screened patients, efficacy was assessed in the following patient numbers (FAS/PPS): total (293/285), TJP-008-1 (98/94), TJP-008-2 (97/95), and 2LPEG (98/96). FAS revealed noninferiority between TJP-008 and 2LPEG with regard to overall success (TJP-0082, 99.0\%; TJP-008-1, 95.9\%; 2LPEG, 94.9\%; $p=0.100$ and $p=0.733$, respectively). PPS also showed noninferiority ( $p=0.721$ and $p=0.211$, respectively). However, the PPS analyses showed a higher bowel cleansing score for TJP-008-2 for high-quality cleansing in the right colon (TJP$008-283.2 \%$ vs 2 LPEG $62.5 \%$; $p=0.005$ ).

Conclusions: TJP-008 is a new low-volume cleansing agent with a colon cleansing efficacy comparable to that of standard 2LPEG that exhibits significant safety and tolerability. (Gut Liver 2022;16:259-268)

Key Words: Colonoscopy; Cathartics; Polyethylene glycols; Ascorbic acid; Safety

\section{INTRODUCTION}

Adequate bowel preparation is critical for the diagnostic and therapeutic success and cost-effectiveness of colonoscopy. ${ }^{1,2}$ Suboptimal cleansing can impede the detection of colorectal neoplasia and lead to early repetitive procedures and intervention delays. ${ }^{3,4}$ Optimal bowel preparation is a prerequisite for adenoma detection. ${ }^{5}$ Excellent bowel cleansing improves detection of sessile serrated polyps. ${ }^{5}$ Because these flat lesions are often found in the ascending colon and cecum and are one of the reasons for cancers di- agnosed after colonoscopy, ${ }^{6}$ effective cleansing of the right colon is important to colorectal cancer prevention.

Polyethylene glycol (PEG)-based agents used with split dosing regimen are considered the gold standard among bowel preparation agents. ${ }^{4,7}$ However, many PEG-based preparations require ingestion of solutions of up to $4 \mathrm{~L}$, which may reduce patient adherence. ${ }^{4,9}$ The addition of ascorbate to the PEG solution allows for the use of a reduced-volume 2 L PEG-based preparation. The laxative effect of ascorbate allows volume reduction of the preparation agent. ${ }^{10}$ PEG with ascorbate has been demonstrated to 
have a high level of efficacy and safety in bowel cleansing for colonoscopy. ${ }^{9,10}$

TJP-008 (CleanViewAL; Taejoon, Seoul, Korea) is a novel 1 L PEG-based bowel preparation agent designed to improve patient adherence and colon cleansing effect by combining PEG with high-dose ascorbate. Herein, we reported the results of our phase 3 trial that compared the effectiveness of TJP-008 and 2 L PEG with ascorbate (2LPEG) in patients undergoing colonoscopy. TJP-008 was administered as a 2-day (evening and morning) split dosing or a 1-day (morning-only) split dosing regimen and 2LPEG as a 2-day split dosing regimen as a standard preparation.

\section{MATERIALS AND METHODS}

\section{Study design}

This study was a randomized, multicenter, investigatormasked, and parallel-group phase 3 clinical trial (ClinicalTrials.gov NCT03544944) and was conducted in six clinical sites. The study was approved by the Institutional Review Board of Korea University Ansan Hospital (IRB number: 2017AS0011) and performed according to the tenets of the Helsinki Declaration and compliance with the International Conference on Harmonization Good Clinical Practice guidelines and Korean regulations. All patients were fully informed and provided written consent for participation before enrollment.

\section{Patients}

Eligible patients were adults over 19 years of age with a body mass index $<30 \mathrm{~kg} / \mathrm{m}^{2}$ undergoing screening, surveillance, or diagnostic colonoscopy. ${ }^{11}$ Women of child-bearing potential were required to have a negative pregnancy test and practice birth control during the study. All patients were outpatients. The primary exclusion criteria included a history of severe constipation, known or suspected ileus, gastrointestinal obstruction, bowel perforation, toxic colitis, megacolon, inflammatory bowel disease, or major colorectal surgeries such as colon resection; abdominal surgery within the previous 6 months; regular use (more than 2 or 3 days a week) of laxatives or colon motilityaltering drugs; active intestinal bleeding; or a clinically significantly low hemoglobin level. Patients with identified or suspected phenylketonuria, severe heart failure (New York Heart Association classification III and IV), or renal insufficiency (creatinine clearance $<30 \mathrm{~mL} / \mathrm{min}$ ) were also excluded.

\section{Treatment allocation and masking}

Patients were randomly assigned to receive TJP-008, administered as a 1-day split dosing (TJP-008-1) or 2-day split dosing (TJP-008-2) regimen, or 2LPEG (Coolprep; Taejoon) agent, administered as a 2-day split dosing regimen.

An independent randomization code generation officer not involved in the trial created a randomization table before the trial initiation. The stratified block randomization method was used to each clinical trial institution to ensure a 1:1:1 allocation across dose groups (TJP-008-1, TJP-0082, and 2LPEG). Participants who met the inclusion criteria at their second visit were then assigned to each dose group using Interactive Web Response System in the order of registration into the study according to the randomization table created using SAS version 9.1 (SAS Institute, Cary, NC, USA). Given that investigational product (TJP-008 and 2LPEG) is supplied in same cartoon box, the investigational drug was managed by dividing the blind part and the unblind part. The investigators were blind part so that they could not know which investigational drug to be administered.

Table 1. TJP-008 and 2LPEG Formulations: Quantities of Osmotically Active Ingredients and Fluid Volumes

\begin{tabular}{|c|c|c|c|c|}
\hline \multirow{2}{*}{ Variable } & \multicolumn{2}{|c|}{ TJP-008 } & \multicolumn{2}{|c|}{ 2LPEG } \\
\hline & Dose 1 & Dose 2 & Dose 1 & Dose 2 \\
\hline \multicolumn{5}{|l|}{ Osmotically active ingredients, g } \\
\hline PEG3350 & 80 & 80 & 100 & 100 \\
\hline Sodium sulfate & 9.0 & 9.0 & 7.5 & 7.5 \\
\hline Sodium ascorbate & 4.7 & 4.7 & 5.9 & 5.9 \\
\hline Ascorbic acid & 20.3 & 20.3 & 4.7 & 4.7 \\
\hline \multicolumn{5}{|l|}{ Electrolytes, g } \\
\hline Sodium chloride & 1.35 & 1.35 & 2.691 & 2.691 \\
\hline Potassium chloride & 0.5 & 0.5 & 1.015 & 1.015 \\
\hline Bowel preparation volume, $\mathrm{mL}$ & 500 & 500 & 1,000 & 1,000 \\
\hline Additional fluid volume, $\mathrm{mL}$ & 500 & 500 & 500 & 500 \\
\hline Total volume, mL & 1,000 & 1,000 & 1,500 & 1,500 \\
\hline
\end{tabular}

2LPEG, $2 \mathrm{~L}$ polyethylene glycol with ascorbate. 


\section{Bowel cleansing schedules and dietary restrictions}

Study treatment compositions are summarized in Table 1. All patients self-administered their bowel preparation agents. Patients in the TJP-008-2 and 2LPEG groups administered the first dose at approximately 18:00 on the day before and the second dose at approximately 06:00 on the day of the colonoscopy (Fig. 1). Patients in the TJP-0081 group administered the first and second doses at 06:00 and 08:00 on the day of the colonoscopy. For maintaining blindness, the colonoscopy time was set to be performed between $10 \mathrm{AM}$ and $1 \mathrm{PM}$ so that both the 1-day split dosing and the 2-day split dosing group can be included. Depending on the colonoscopy time and the group, each patient was given time to take a bowel cleansing agent. A window of \pm 2 hours from the start time was allowed. For all patients, bowel preparations were usually conducted at home.

On the day before colonoscopy, patients had a light breakfast (all groups) and a light lunch (all groups), followed by a low-fiber diet for dinner (TJP-008-1) or no dinner (TJP-008-2 and 2LPEG groups). Patients were not allowed to consume additional clear fluids from the start of the first dose for colonoscopy until full recovery from sedation. To ensure adequate colon cleansing and the correct use of colon cleansing agents, patients were educated on how to take a colon cleansing agent according to the patient groups and what foods to avoid before taking the agent through the dosing guide.

\section{Assessments and endpoints}

Clinical decisions were guided by site colonoscopists who have performed colonoscopy for at least 3 years in an educational hospital. Therefore, experienced site colonoscopists performed all colonoscopy and assigned initial segmental cleansing scores using the Harefield Cleansing Scale (HCS) (Fig. 2). ${ }^{11}$ Inter-colonoscopist assessment variability was minimized by using a guideline including the video recording method and measurements of bowel cleansing in each colonoscopy. Overall HCS grades (success=A or B; failure $=\mathrm{C}$ or $\mathrm{D}$ ) were computed from five segmental scores
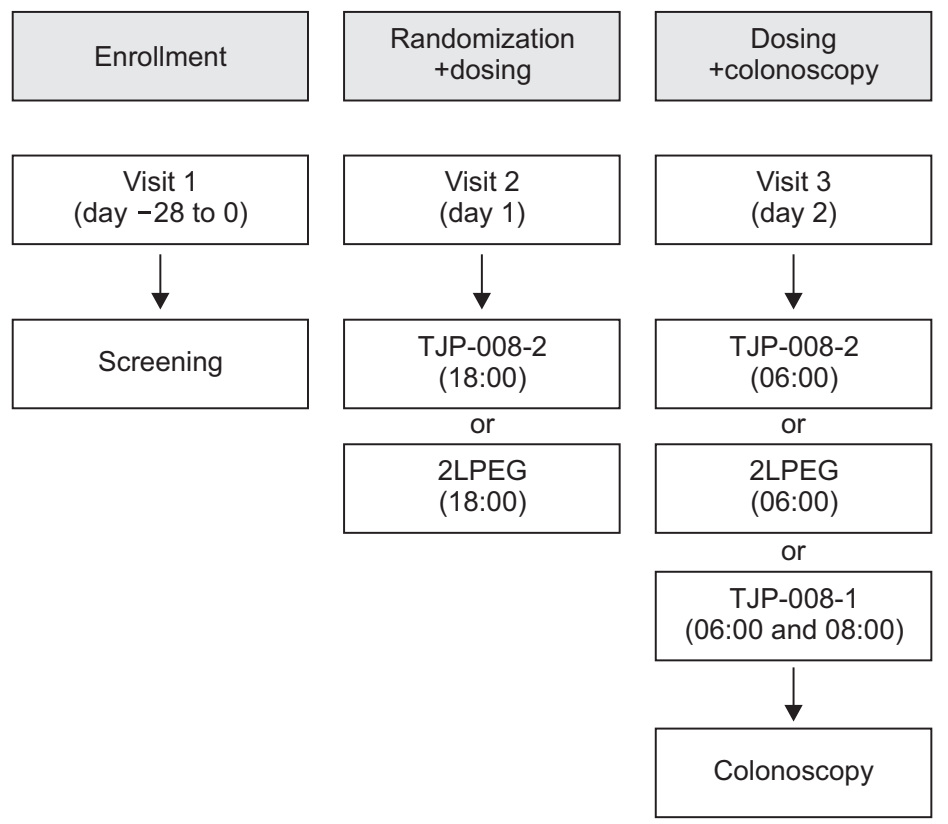
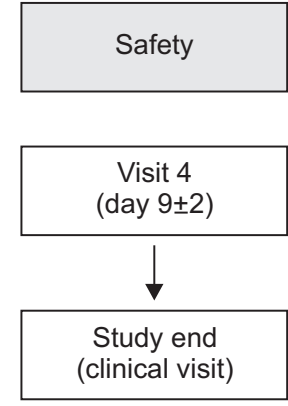

(clinical visit)
Fig. 1. Study protocol. TJP-008-2, TJP-008 administered as an evening/morning regimen; TJP008-1, TJP-008 administered as a morning-only regimen; 2LPEG, 2 L polyethylene glycol with ascorbate.
Image

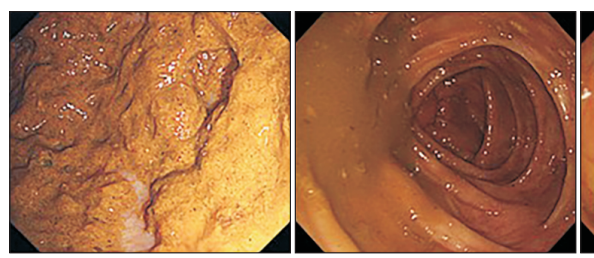

Score 0

Irremovable,

score and

description

Semi-solid only
partially

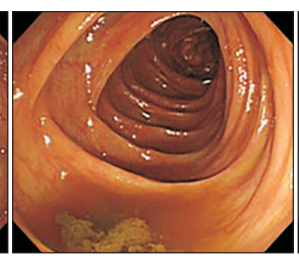

Score 2

Score 1

Brown

removable stools liquid/removable

semi-solid stools

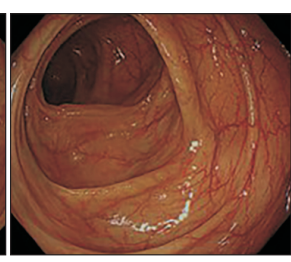

Score 4

Empty and clean

Fig. 2. Sample endoscopic images for segmental scoring using the Harefield Cleansing Scale (HCS). 
of $0-4 .^{12,13}$ The primary end point was the overall colon cleansing effect as measured by the overall HCS grade.

Secondary end points included a high-quality bowel cleansing rate (score 4 and 3 on the HCS) in each colon segment and a polyp detection rate (PDR) in overall colon and the right colon (assessed by the on-site colonoscopist), adenoma detection rate (ADR) in the overall colon and the right colon (assessed through a pathological review of polyp biopsies). Lesion detection rates were calculated as the percentage of patients with at least one adenoma (for ADR) or polyp (for PDR) in the analyzed population.

Tolerability, acceptability, and adherence were assessed before colonoscopy. Taking more than $75 \%$ of each dose was defined as successful adherence. ${ }^{14,15}$ The dose was confirmed through the patient diary provided to the patient at visit 2 . Tolerability and acceptability were inves- tigated using a patient survey on the day of colonoscopy.

The safety was evaluated based on the incidence and characteristics of adverse events developed after administration of bowel cleansing agents, physical parameters, vital signs, electrocardiogram, and clinical laboratory findings related to taking cleansing agents.

\section{Sample size calculation}

This study is primarily intended to demonstrate the noninferiority of 1 L PEG (TJP-008) to 2LPEG (control) in terms of colon cleaning efficacy. When the maximum value of the confidence intervals was less than $15 \%$, the trial groups were judged to be not inferior to the control group. The upper limit of noninferiority was set at $15 \%$ as it was the standard acceptable level for noninferiority in previous bowel preparation studies. ${ }^{9,16,17}$ Assuming overall cleans-

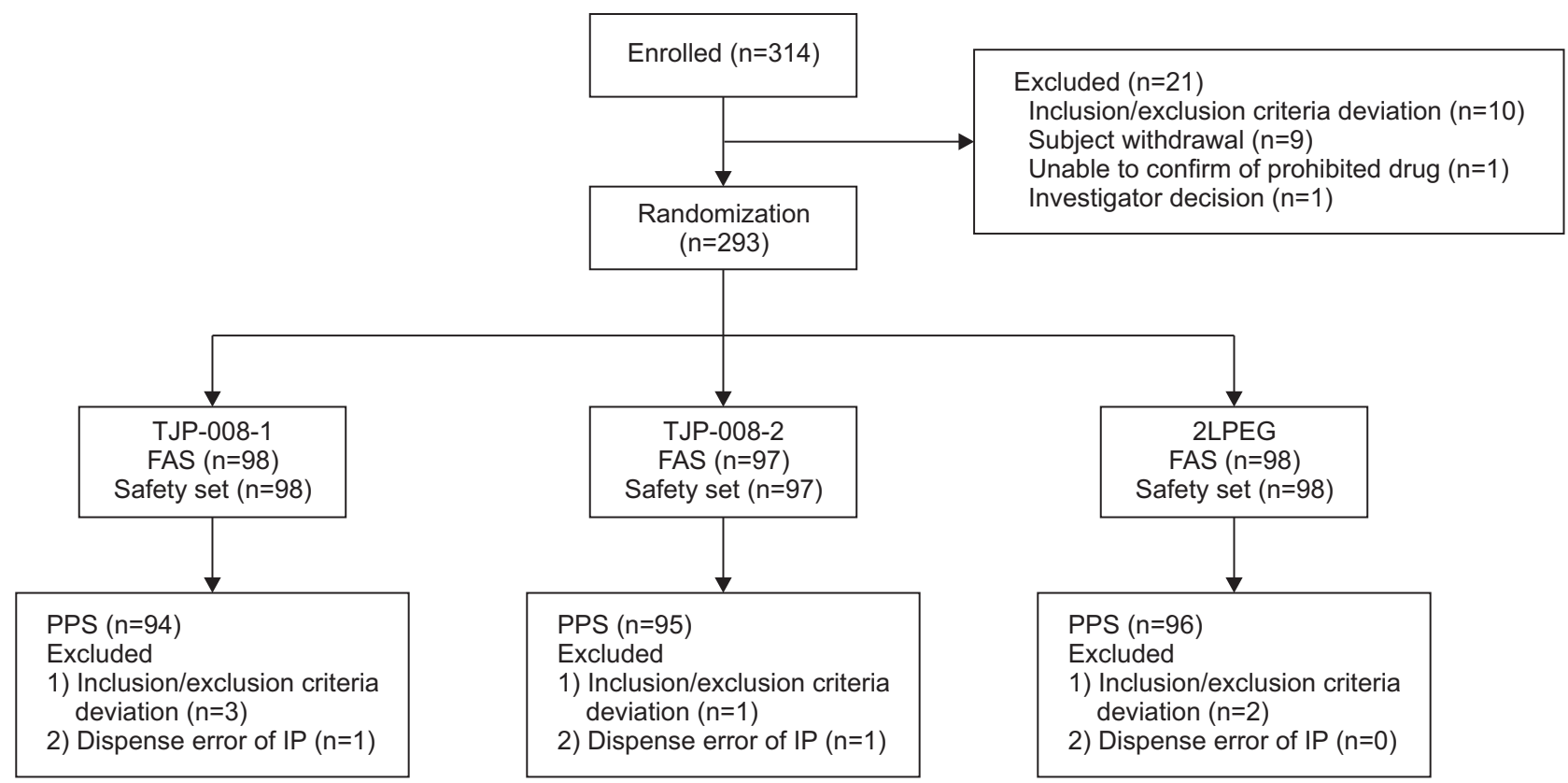

Fig. 3. Patient flowchart.

TJP-008-2, TJP-008 administered as an evening/morning regimen; TJP-008-1, TJP-008 administered as a morning-only regimen; 2LPEG, 2 L polyethylene glycol with ascorbate; FAS, full analysis set; PPS, per protocol set; IP, investigational product.

Table 2. Baseline Characteristics of the Enrolled Patients (Full Analysis Set)

\begin{tabular}{lccc}
\hline \multicolumn{1}{c}{ Characteristics } & TJP-008-2 (n=97) & TJP-008-1 (n=98) & 2LPEG (n=98) \\
\hline Age, mean (range), yr & $48.0(25-78)$ & $49.5(22-75)$ & $45.5(21-77)$ \\
Male sex, No. (\%) & $35(37.1)$ & $45(45.9)$ & $41(41.8)$ \\
BMI, mean \pm SD, $\mathrm{kg} / \mathrm{m}^{2}$ & $24.16 \pm 2.91$ & $24.44 \pm 2.86$ & $24.09 \pm 2.68$ \\
Screening colonoscopies, No. (\%) & $28(28.9)$ & $38(38.8)$ & $43(43.9)$ \\
Known renal and urinary disorders, No. (\%) & $2(2.1)$ & $1(1.0)$ & 0 \\
$\quad$ Bladder diverticulum & $1(1.0)$ & 0 & 0 \\
Chronic kidney disease & 0 & $1(1.0)$ & 0 \\
Neurogenic bladder & $1(1.0)$ & 0 & 0 \\
\hline
\end{tabular}

TJP-008-2, TJP-008, administered as an evening/morning regimen; TJP-008-1, TJP-008, administered as a morning-only regimen; 2LPEG, 2 L polyethylene glycol with ascorbate; BMI, body mass index. 
ing success rates of $88.9 \%{ }^{9}$ for all three groups, and with a noninferiority margin of $15 \%$ and a one-sided significance threshold of $\mathrm{p}<0.025$, we assessed a sample of 79 patients per group, which provided a statistical power of at least $85 \%$ to demonstrate noninferiority. Finally, 99 patients per group were investigated considering the dropout rate of $20 \%$.

\section{Statistical analysis}

Statistical analyses were conducted using SAS version 9.2 (SAS Institute, Cary, NC, USA). An alpha value of 0.05 was split across the primary end point, providing a onesided significance threshold of $\mathrm{p}<0.025$. To compare the two TJP-008 groups with the control group, we first evalu- ated TJP-008-2 using a gatekeeping approach and then TJP-008-1 if successful (each vs 2LPEG). The bowel cleaning successful rate were evaluated through the chi-square tests or the Fisher exact tests. Additionally, if there were any statistically significant variables among sex, ages, and body mass index distribution, an analysis of covariance was performed to correct for these variables as a sensitivity analysis. Secondary end points were tested through the following steps: ADR in the right colon; ADR in the overall colon; PDR in the right colon; PDR in the overall colon. Tolerability was evaluated with the percentage in each group and those differences between the groups were evaluated through chi-square tests or Fisher exact tests.

The full analysis set (FAS) contained all randomized
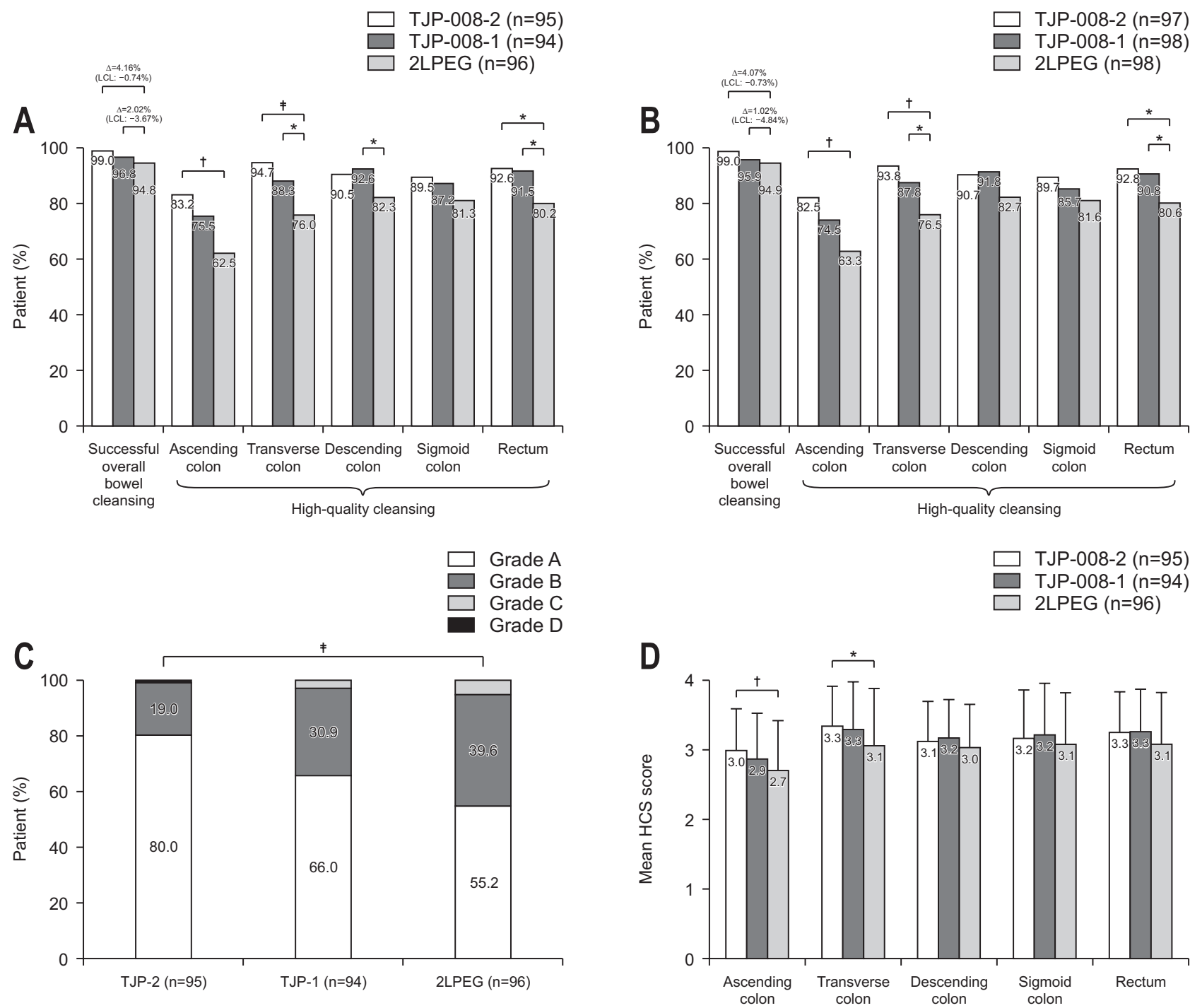

Fig. 4. Bowel cleansing efficacy according to (A) the Harefield Cleansing Scale (HCS) in the per protocol set, (B) the HCS in the full analysis set, (C) the HCS grades in the per protocol set, and (D) mean segmental HCS scores in the per protocol set.

$\mathrm{LCL}$, one-sided $97.5 \%$ lower confidence limit for the difference between treatments; $\Delta$, difference in rate; TJP-008-2, TJP-008 administered as an evening/morning regimen; TJP-008-1, TJP-008 administered as a morning-only regimen; 2LPEG, 2 L polyethylene glycol with ascorbate. * $p<0.05$, ${ }^{\dagger} p<0.01,{ }^{\ddagger} p<0.001$. 
patients who were enrolled after the screening test in the study. The per protocol set (PPS) included patients without major protocol deviations, who met eligibility criteria, who took $\geq 75 \%$ of each dose, and who had available data for the primary end point. Missing efficacy data were replaced as failures. The safety set consisted of all patients for whom the possibility of having received more than one study medication could not be ruled out (based on patient survey).

\section{RESULTS}

\section{Patient characteristics}

Patients were enrolled between January and April 2018, with follow-up completed in May 2018. Among total 314 patients enrolled in the screening test, after excluding 21 patients (screening failure, 10 patients; withdrawal of consent, 9 patients; uncertain drug history, 2 patients), 293 were randomized and comprised the FAS. Among them, 285 (97.3\%) were included in the PPS (Fig. 3). The most common reason for exclusion from the FAS was deviation from the inclusion/exclusion criteria $(n=6)$. The FAS included each of the patients in the TJP-008-1 and TJP-008-2 groups, among whom dispense errors of the investigational product were imputed as failures.

Overall, demographic characteristics were well balanced between the groups (Table 2). In the FAS, similar proportions of patients in the TJP-008-1, TJP-008-2, and 2LPEG groups underwent screening colonoscopy $(28.9 \%$ to $43.9 \%)$.

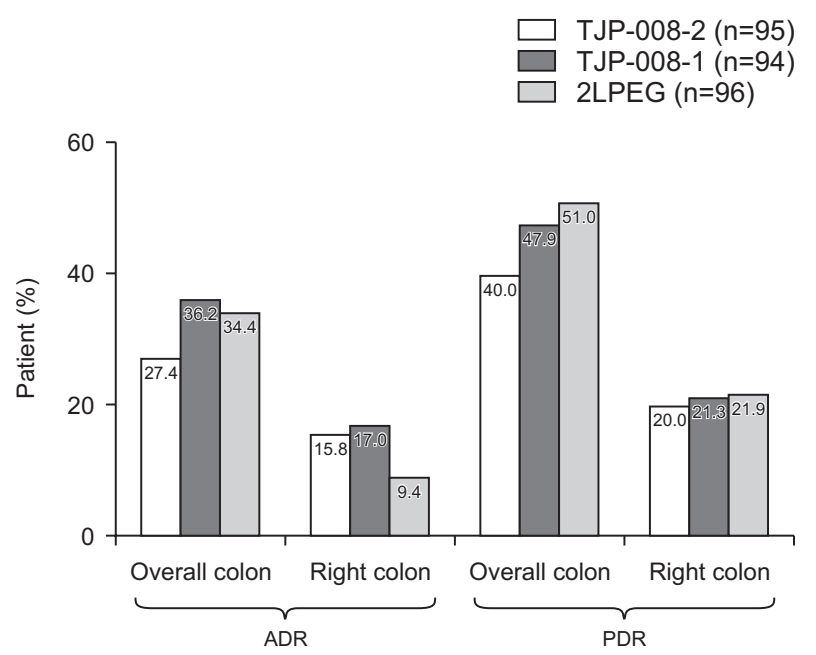

Fig. 5. Lesion detection (per protocol set).

ADR, adenoma detection rate; PDR, polyp detection rate; TJP-008-2, TJP-008 administered as an evening/morning regimen; TJP-008-1, TJP-008 administered as a morning-only regimen; 2LPEG, $2 \mathrm{~L}$ polyethylene glycol with ascorbate.

\section{Bowel cleansing efficacy}

\section{1) Overall colon}

Both TJP-008 regimens achieved noninferiority versus 2LPEG for the primary end point of successful overall bowel cleansing (Fig. 4A). High success rates of overall bowel cleansing were achieved in all three groups of PPS (TJP-008-2, 99.0\%; TJP-008-1, 96.8\%; 2LPEG, 94.8\%). TJP-008-2 and TJP-008-1 were noninferior to 2LPEG for overall bowel cleansing (lower confidence interval for the difference vs 2 LPEG: $-0.74 \%$ and $-3.67 \%$, respectively). In FAS, high rates of overall bowel cleansing success were achieved in all groups, and both TJP-008 groups also revealed noninferiority to 2LPEG (TJP-008-2, 99.0\%; TJP008-1, 95.9\%; 2LPEG, 94.9\%) (Fig. 4B).

In the PPS, the proportion of HCS grades were significantly different between the TJP-008-2 and 2LPEG groups $(\mathrm{p}<0.001)$, although the proportion in the TJP-008-1 group was not significantly different ( $\mathrm{p}=0.290$ ) (Fig. 4C).

\section{2) Colon segments}

High-quality bowel preparation in the right colon was achieved in $83.2 \%$ (79/95) of patients in the TJP-008-2 group, 75.5\% (71/94) of patients in the TJP-001-1 group, and $62.5 \%(60 / 96)$ of patients in the 2LPEG group (Fig. 4A). Noninferiority was observed for the TJP-008-2 and TJP-008-1 compared to the 2LPEG group. Furthermore, high-quality cleansing rates were significantly higher in the TJP-008-2 group than in the 2LPEG group ( $\mathrm{p}=0.001)$. In the right colon, the mean segmental score of high-quality cleansing significantly improved in the TJP-008-2 group, although no significant difference was observed between

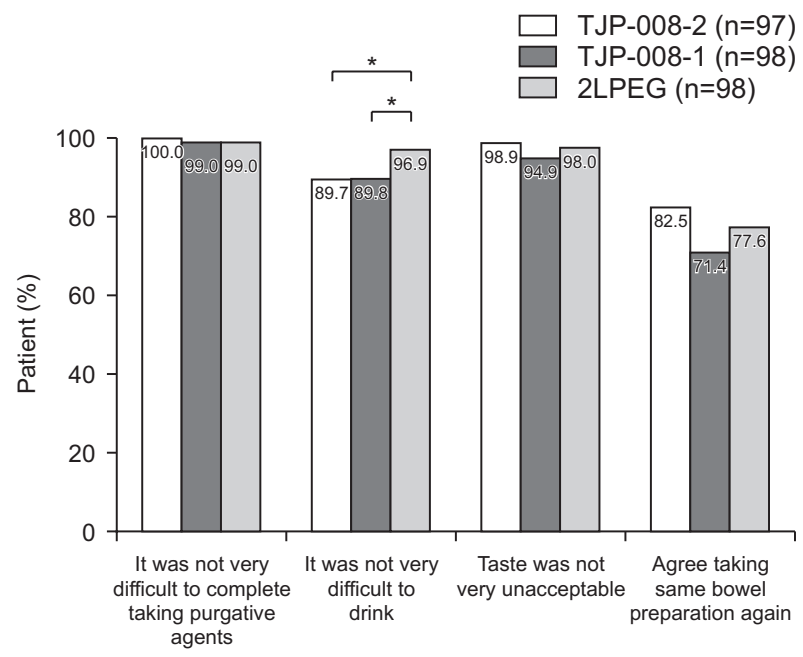

Fig. 6. Tolerance and acceptability of bowel preparations among respondents of the patient survey (safety set).

TJP-008-2, TJP-008 administered as an evening/morning regimen; TJP-008-1, TJP-008 administered as a morning-only regimen; 2LPEG, $2 \mathrm{~L}$ polyethylene glycol with ascorbate. ${ }^{*} p<0.05$. 
the TJP-008-1 and 2LPEG groups (Fig. 4D).

Among other colon segments except the ascending colon, two TJP-008 groups displayed noninferiority to the 2LPEG group and even significantly higher-quality cleansing rates than the 2LPEG group in the two bowel segments (transverse colon and rectum; $\mathrm{p}<0.05$ for both) (Fig. 4A). Similarly, mean segmental cleansing scores in other colon segments of the TJP-008 groups were not inferior to those of the 2LPEG group (Fig. 4D).

\section{Lesion detection}

The ADR and PDR in the right colon and in the overall colon in the TJP-008-1 and TJP-008-2 groups were not significantly different to those in the 2LPEG group in the PPS (Fig. 5). In the right colon, the ADR was $17.0 \%$ and $15.8 \%$ for the TJP-008-1 and TJP-008-2 groups, respectively, compared to $9.4 \%$ for $2 \mathrm{LPEG}$ ( $\mathrm{p}=0.162$ and $\mathrm{p}=0.221$, respectively). The overall colon $\mathrm{ADR}$ was $36.2 \%$ for the TJP-008-01 group and 27.4\% for the TJP-008-2 group when compared to $34.4 \%$ for the 2LPEG group $(p=0.903$ and $\mathrm{p}=0.362$, respectively). The PDRs in the right colon of both TJP-008 groups were not significantly different from that in the 2LPEG group (21.3\% and $20.0 \%$ vs $21.9 \%$, respectively). The PDR in the overall colon was $47.9 \%$ for the TJP-008-1 group and 40.0\% for the TJP-008-2 group, compared to $51.0 \%$ for the 2LPEG group ( $\mathrm{p}=0.680$ and $\mathrm{p}=0.238$, respectively).

\section{Tolerability, acceptability, and adherence}

As revealed from patient survey responses, tolerability and acceptability of the bowel preparations, except for difficulty in drinking the treatment agents, were similar among the three treatment groups (Fig. 6). The self-reported adherence rate was $100 \%$ across all treatment groups in both FAS and PPS.

\section{Safety}

Treatment-emergent adverse events (TEAEs) that were considered treatment-related were reported among 15.3\%, $16.5 \%$, and $8.2 \%$ of patients in the TJP-008-1, TJP-0082 , and 2LPEG groups of safety set, respectively $(\mathrm{p}=0.179)$. These TEAEs were mild in severity and gastrointestinal in nature (Table 3 ). The most frequent TEAE for the TJP-0082 group was nausea; TJP-008-1, thirst; 2LPEG, vomiting. Vomiting rates were $1.0 \%$ for the 2LPEG group; TJP-008$1,3.1 \%$ ( $\mathrm{p}=0.621$ vs $2 \mathrm{LPEG}$ ); TJP-008-2, $6.2 \%$ ( $\mathrm{p}=0.065$ vs 2LPEG). Nausea rates were $3.1 \%$ for the TJP-008-1 group and $2.1 \%$ for the TJP-008-02 group. Abdominal pain, including the epigastric pain, occurred in one patient each in TJP-008-1 group and 2LPEG group. However, no patient discontinued pharmacotherapy because of treatmentrelated TEAEs. No deaths or related serious TEAEs were reported.

On physical examination and electrocardiogram, there were no clinically significant findings after the cleansing agents. In vital signs, one in the TJP-008-1 group and one in the TJP-008-02 group reported abnormal findings of clinically significant blood pressure after colonoscopy (visit 3). All recovered at the follow-up visit.

In general, median changes from the baseline in hematologic, clinical chemistry, and urinalysis parameters were not considered clinically significant, and no clinically significant differences were observed between groups. There were no clinically significant changes in all laboratory findings, including renal function, even in patients over 65 years of age. The incidence of electrolyte shifts from normal at baseline to high at post-baseline visits are summarized in Table 4.

Table 3. Safety Summary (Safety Set)

\begin{tabular}{|c|c|c|c|c|c|}
\hline \multirow{2}{*}{ Variable } & \multicolumn{2}{|c|}{ TJP-008-2 (n=97) } & \multicolumn{2}{|c|}{ TJP-008-1 (n=98) } & \multirow{2}{*}{$\begin{array}{c}\text { 2LPEG (n=98), } \\
\text { No. }(\%)\end{array}$} \\
\hline & No. $(\%)$ & p-value* & No. $(\%)$ & $p$-value ${ }^{+}$ & \\
\hline Total number of treatment-related TEAEs & 16 & & 18 & & 11 \\
\hline Patients with treatment-related TEAEs & $16(16.5)$ & 0.287 & 15 (15.3) & 0.159 & $8(8.2)$ \\
\hline \multicolumn{6}{|l|}{ Patients with specific treatment-related TEAEs ${ }^{\ddagger}$} \\
\hline Vomiting & $6(6.2)$ & 0.065 & $3(3.1)$ & 0.621 & $1(1.0)$ \\
\hline Nausea & $2(2.1)$ & NA & $3(3.1)$ & NA & 0 \\
\hline Thirst & $2(2.1)$ & 0.621 & $4(4.1)$ & 0.369 & $1(1.0)$ \\
\hline Patients with treatment-related severe TEAEs & 0 & & 0 & & 0 \\
\hline Patients with treatment-related serious TEAEs & 0 & & 0 & & 0 \\
\hline Deaths & 0 & & 0 & & 0 \\
\hline
\end{tabular}

TJP-008-2, TJP-008 administered as an evening/morning regimen; TJP-008-1, TJP-008 administered as a morning-only regimen; 2LPEG, 2 L polyethylene glycol with ascorbate; TEAE, treatment-emergent adverse event; NA, not available.

${ }^{*}$ TJP-008-2 vs 2LPEG: Pearson chi-square test or Fisher exact test; ${ }^{\dagger}$ TJP-008-1 vs 2LPEG: Pearson chi-square test or Fisher exact test; ${ }^{\ddagger}$ Reported for at least $2 \%$ of patients in any treatment group. 
Table 4. Incidence of Shifts in Electrolytes from Normal* at Screening Test to High at Post-Screening Visits (Safety Set)

\begin{tabular}{lccc}
\hline \multicolumn{1}{c}{ Variable } & TJP-008-2 (n=97) & TJP-008-1 (n=98) & 2LPEG (n=98) \\
\hline Sodium & & & \\
$\quad$ Visit 2 (baseline) & 0 & $1(1.0)$ & 0 \\
Visit 3 (colonoscopy day) & $4(4.2)$ & 0 & 0 \\
Visit 4 (7 days after colonoscopy) & 0 & & 0 \\
Potassium & $6(1.0)$ & $3(3.1)$ \\
Visit 2 (baseline) & $11(11.3)$ & $1(1.0)$ & 0 \\
Visit 3 (colonoscopy day) & $2(2.1)$ & $17(17.3)$ & $2(2.0)$ \\
Visit 4 (7 days after colonoscopy) & $9(1.0)$ & $5(5.1)$ \\
Chloride & $51(52.6)$ & $4(4.1)$ & $15(15.3)$ \\
Visit 2 (baseline) & $6(6.2)$ & $47(48.0)$ & $7(7.1)$ \\
Visit 3 (colonoscopy day) & & $4(4.1)$ & \\
Visit 4 (7 days after colonoscopy) & & \\
\hline
\end{tabular}

Data are presented as the number (\%).

TJP-008-2, TJP-008 administered as an evening/morning regimen; TJP-008-1, TJP-008 administered as a morning-only regimen; 2LPEG, 2 L polyethylene glycol with ascorbate.

*The upper limit of normal: sodium, $145 \mathrm{mmol} / \mathrm{L}$; potassium, $5 \mathrm{mmol} / \mathrm{L}$; chloride, $105 \mathrm{mmol} / \mathrm{L}$.

\section{DISCUSSION}

In the PPS population, both TJP-008 regimens were noninferior to the standard 2LPEG regimen in overall bowel cleansing. As shown in Fig. 4, overall cleansing success rates measured using the HCS were high in all three groups. TJP-008-2 and TJP-008-1 achieved successful bowel cleansing rates of $99.0 \%$ and $96.8 \%$, respectively, among patients, which were higher than those reported in some previous studies on PEG-based preparation agents ${ }^{9,16,17}$ and the minimum criteria for adequate bowel cleansing recommended by the European Society of Gastrointestinal Endoscopy. ${ }^{18}$ In the FAS, both TJP-008 groups also demonstrated noninferior bowel cleansing efficacy with the 2LPEG regimen. Although TJP-008-1 was similar to 2LPEG in the proportion of HCS grades, TJP-008-2 displayed a significantly different proportion.

Finally, both TJP-008 dosing regimens achieved segmental high-quality cleansing noninferiority on the HCS in right colon and other colon segments versus 2LPEG. TJP-008-2 regimen displayed superior high-quality cleansing in the right colon vs 2 LPEG. This superiority on right colon was also observed in the FAS populations. Therefore, TJP-008 markedly increases the ADR, which is associated with a reduction in colorectal cancer. ${ }^{19-22}$

Improvement of right-sided colon cleansing with TJP008 may help detect high risk sessile serrated polyps, which can be better detected in higher-quality bowel preparations. ${ }^{5}$ Although this study did not assess directly sessile serrated polyps, TJP-008-2 displayed superior cleansing and superior tendency of ADR in the right colon versus 2LPEG. An increase in the osmotic activity of TJP-008 due to an increase in ascorbic acid levels may be associated with high cleansing levels in the right colon.
All three groups displayed complete adherence with intake for both the bowel preparation agents and recommended additional clear fluids in the self-reported patient survey. Although it was more difficult to drink TJP-008, all cleansing regimens were generally well accepted and tolerated.

The overall safety profile of TJP-008 was similar to that of 2LPEG. Treatment-related TEAEs were generally transient and mild in severity, reflecting the expected safety profiles of PEG-based bowel preparations. Although vomiting, nausea, and thirst were more common with TJP-008, these rates were low, and no effect was observed on efficacy or adherence. These adverse reactions may be associated with a transient electrolyte imbalance during bowel cleansing. ${ }^{23}$

Consistent efficacy, acceptability, adherence, and safety between the two TJP-008 regimens (1-day and 2-day split dose) support the flexible dosing in accordance with the physician and patient preferences and the intended timing of colonoscopy.

This study has potential limitations. The sample size was not large and the noninferiority margin was $15 \%$ in this study, although there was no significant difference between the TJP-008 groups and the 2LPEG group in the colon cleansing effect. The inclusion of patients with prior colonoscopy ( $70 \%$ of the FAS) may limit comparisons with studies focused on patients with screening colonoscopy and affect subjective evaluation of the bowel preparation agents. A self-reported patient survey may not be as reliable as validated patient-reported outcome questionnaires or objective measures. Our results may not be generalizable to patients with severe constipation or a high body mass index $\left(>30 \mathrm{~kg} / \mathrm{m}^{2}\right)$, who were excluded from this study. 
The study has some strengths. The study had a randomized, multicenter design. Although the individual colonoscopists evaluated cleansing efficacy using a validated cleansing scale, all site colonoscopists were blind to the treatment assignments. Both primary and secondary end points for bowel cleansing effects were evaluated using the HCS. The study findings are also clinically relevant. Highquality preparations based on the HCS may facilitate endoscopic procedure and high-quality colonoscopy. Therefore, the higher cleansing efficacy observed with the new lowervolume preparation is associated with a higher-quality colonoscopy among patients.

Among patients undergoing colonoscopy, the new 1 L PEG-based bowel preparation, TJP-008 displayed high bowel cleansing efficacy, which was also well accepted for flexible 2-day (evening and morning) split dosing or 1-day (morning-only) split dosing. The TJP-008 treatment regimens resulted in higher rates of segmental high-quality cleansing, and potentially reduced the need for additional cleaning during colonoscopy.

\section{CONFLICTS OF INTEREST}

No potential conflict of interest relevant to this article was reported.

\section{ACKNOWLEDGEMENTS}

The authors would like to thank the principal investigators from the six institutions for their expertise.

\section{AUTHOR CONTRIBUTIONS}

Study design: Y.T.J. Data collection: J.S.K., J.S.B., B.I.L., S.A.J., T.I.K., Y.T.J. Data analysis: J.S.K. Drafting of the manuscript: J.S.K. Revising the manuscript for important intellectual content: J.S.B., B.I.L., S.A.J., T.I.K. Study supervision: Y.T.J. All authors have read and approved the final version to be published.

\section{ORCID}

Ja Seol Koo https://orcid.org/0000-0002-1202-075X Jeong-Sik Byeon https://orcid.org/0000-0002-9793-6379 Bo-In Lee https://orcid.org/0000-0002-0218-4136 Sung-Ae Jung https://orcid.org/0000-0001-7224-2867 Tae Il Kim https://orcid.org/0000-0003-4807-890X
Yoon Tae Jeen

https://orcid.org/0000-0003-0220-3816

1. Levin B, Lieberman DA, McFarland B, et al. Screening and surveillance for the early detection of colorectal cancer and adenomatous polyps, 2008: a joint guideline from the American Cancer Society, the US Multi-Society Task Force on Colorectal Cancer, and the American College of Radiology. Gastroenterology 2008;134:1570-1595.

2. Belsey J, Epstein O, Heresbach D. Systematic review: oral bowel preparation for colonoscopy. Aliment Pharmacol Ther 2007;25:373-384.

3. Menees SB, Elliott E, Govani S, et al. The impact of bowel cleansing on follow-up recommendations in average-risk patients with a normal colonoscopy. Am J Gastroenterol 2014;109:148-154.

4. Hassan C, East J, Radaelli F, et al. Bowel preparation for colonoscopy: European Society of Gastrointestinal Endoscopy (ESGE) Guideline. Update 2019. Endoscopy 2019;51:775794.

5. Clark BT, Laine L. High-quality bowel preparation is required for detection of sessile serrated polyps. Clin Gastroenterol Hepatol 2016;14:1155-1162.

6. Rex DK, Ahnen DJ, Baron JA, et al. Serrated lesions of the colorectum: review and recommendations from an expert panel. Am J Gastroenterol 2012;107:1315-1329.

7. Bechtold ML, Mir F, Puli SR, Nguyen DL. Optimizing bowel preparation for colonoscopy: a guide to enhance quality of visualization. Ann Gastroenterol 2016;29:137-146.

8. Belsey J, Crosta C, Epstein O, et al. Meta-analysis: the relative efficacy of oral bowel preparations for colonoscopy 19852010. Aliment Pharmacol Ther 2012;35:222-237.

9. Ell C, Fischbach W, Bronisch HJ, et al. Randomized trial of low-volume PEG solution versus standard PEG + electrolytes for bowel cleansing before colonoscopy. Am J Gastroenterol 2008;103:883-893.

10. Worthington J, Thyssen M, Chapman G, Chapman R, Geraint M. A randomised controlled trial of a new 2 litre polyethylene glycol solution versus sodium picosulphate + magnesium citrate solution for bowel cleansing prior to colonoscopy. Curr Med Res Opin 2008;24:481-488.

11. Fayad NF, Kahi CJ, Abd El-Jawad KH, et al. Association between body mass index and quality of split bowel preparation. Clin Gastroenterol Hepatol 2013;11:1478-1485.

12. Halphen M, Heresbach D, Gruss HJ, Belsey J. Validation of the Harefield Cleansing Scale: a tool for the evaluation of bowel cleansing quality in both research and clinical practice. Gastrointest Endosc 2013;78:121-131.

13. Parmar R, Martel M, Rostom A, Barkun AN. Validated 
scales for colon cleansing: a systematic review. Am J Gastroenterol 2016;111:197-204.

14. Bisschops R, Manning J, Clayton LB, Ng Kwet Shing R, Álvarez-González M; MORA Study Group. Colon cleansing efficacy and safety with 1 L NER1006 versus 2 L polyethylene glycol + ascorbate: a randomized phase 3 trial. Endoscopy 2019;51:60-72.

15. Schreiber S, Baumgart DC, Drenth JPH, et al. Colon cleansing efficacy and safety with 1 L NER1006 versus sodium picosulfate with magnesium citrate: a randomized phase 3 trial. Endoscopy 2019;51:73-84.

16. Matro R, Shnitser A, Spodik M, et al. Efficacy of morningonly compared with split-dose polyethylene glycol electrolyte solution for afternoon colonoscopy: a randomized controlled single-blind study. Am J Gastroenterol 2010;105:1954-1961.

17. Rex DK, Di Palma JA, Rodriguez R, McGowan J, Cleveland $\mathrm{M}$. A randomized clinical study comparing reduced-volume oral sulfate solution with standard 4-liter sulfate-free electrolyte lavage solution as preparation for colonoscopy. Gastrointest Endosc 2010;72:328-336.

18. Kaminski MF, Thomas-Gibson S, Bugajski M, et al. Perfor- mance measures for lower gastrointestinal endoscopy: a European Society of Gastrointestinal Endoscopy (ESGE) Quality Improvement Initiative. Endoscopy 2017;49:378-397.

19. Corley DA, Jensen CD, Marks AR, et al. Adenoma detection rate and risk of colorectal cancer and death. N Engl J Med 2014;370:1298-1306.

20. Kaminski MF, Wieszczy P, Rupinski M, et al. Increased rate of adenoma detection associates with reduced risk of colorectal cancer and death. Gastroenterology 2017;153:98105.

21. Lasisi F, Rex DK. Improving protection against proximal colon cancer by colonoscopy. Expert Rev Gastroenterol Hepatol 2011;5:745-754.

22. Pontone S, Hassan C, Maselli R, et al. Multiple, zonal and multi-zone adenoma detection rates according to quality of cleansing during colonoscopy. United European Gastroenterol J 2016;4:778-783.

23. Adamcewicz M, Bearelly D, Porat G, Friedenberg FK. Mechanism of action and toxicities of purgatives used for colonoscopy preparation. Expert Opin Drug Metab Toxicol 2011;7:89-101. 